\title{
IMPROVED METHODS FOR CORRELATING TRANSFORMS IN MULTIPLE DESCRIPTION CODING SYSTEMS
}

\author{
Claus Bauer, Corey Cheng \\ Dolby Laboratories, San Francisco, USA, $\{\mathrm{cb}, \mathrm{cnc}\} @$ dolby.com
}

\begin{abstract}
Multiple description has been widely investigated as a quality of service technology for the delivery of multimedia information over lossy channels. This paper presents generalized methods for transform based multiple description that do not introduce additional distortion to the transformed information. Previous noiseless methods place restrictions on how the information may be quantized prior to correlation. Typically, the information must be quantized with quasi-uniform quantizers which all have the same quantization step size. This paper expands these methods so that the information may be quantized with any quantizer, and different parts of the information may be quantized with different quantization step sizes. For specific correlating transforms, we propose a computationally efficient correlation transform that makes use of the Hadamard matrix.
\end{abstract}

\section{INTRODUCTION}

Multiple description has been widely researched as a quality of service technology for sending information over lossy networks. In particular, correlating transform based methods [1], [2], [3] transform information into multiple, statistically correlated subsets of information, called descriptions, and these multiple descriptions are sent over different paths or channels from the sender to the receiver. If the receiver receives all descriptions, the content can be perfectly reconstructed at the sender. If only a subset of the descriptions is received, the receiver uses the statistical correlation among the received descriptions to estimate the content of the missing descriptions.

We review the principles of transform based multiple description as discussed in [1], [2]. Let $x=\left(x_{1}, . ., x_{N}\right)^{t r}$ be a set of $N$ transform coefficients that are all quantized by the same uniform quantizer $Q$ ([4]) with the same quantization step size $Q_{\triangle}$. The $x_{i}$ represent a specific part of the signal representation, e.g. a frequency presentation of an audio frame. Let there be $G$ descriptions, and let the coefficients $x_{i}$ be divided into $N / G$ groups. In each group, the $G$ coefficients are transformed into a set of new coefficients $y=\left(y_{1}, \ldots, y_{G}\right)^{t r}$, where we call the elements of $y$ multiple description $(M D)$ coefficients. The $M D$ coefficients $y$ are independently entropy encoded and sent over the network, possibly using diverse channels to ensure that description erasures are not concurrent.
Following [1], we define a transform $F_{T, Q}(x)=y$ via an $G \times G$ matrix $T$ with determinant equal to one and via the quantizer $Q$. It is known that $T$ can be decomposed in at least one way as $T=T_{1} \ldots T_{k}$ where each matrix $G \times G T_{i}$ has unit entries at the diagonal and nonzero off-diagonal elements in only one row or column. We define

$$
y=F_{T, Q}(x)=Q^{v}\left(T_{1} Q^{v}\left(T_{2} \ldots Q^{v}\left(T_{k} x\right)\right)\right) .
$$

Here, $Q^{v}(z)$ is a vector which quantizes each component of $z \in \mathbb{R}^{G}$ with the quantizer $Q$ and the same quantization step size $Q_{\triangle}$. In [1], [2] it is shown that the quantized coefficients $x$ can be exactly recovered at the decoder from a decorrelating transform if all of the MD coefficients are received:

$$
x=F_{T^{-1}, Q}(y)=Q\left(T_{k}^{-1} \ldots Q\left(T_{2}^{-1} \ldots Q\left(T_{1}^{-1} y\right)\right)\right) .
$$

As the transforms $F_{T, Q}$ and $F_{T^{-1}, Q}$ do not add any distortion to the signal, we call them noiseless transforms. We note that in this scheme, all quantization based distortion is due to the quantization of the transform coefficients that takes place prior to the correlation transform.

If some of the $M D$ coefficients are lost, they are estimated from the received $M D$ coefficients by calculating the conditional expectation for the missing $M D$ coefficients given the received $M D$ coefficients. This estimation requires the knowledge of the variances - measured over time - of the coefficients $x_{i}$ at the sender. Therefore, the variances are sent as side information with the $M D$ coefficients to the sender.

An analysis of the proofs in [1] shows that the noiseless recovery of the coefficients $x$ from the transforms $F_{T, Q}$ and $F_{T^{-1}, Q}$ requires that for any real number $x_{1}$ and $x_{2}$, the quantizer $Q$ satisfies the following conditions:

$$
\begin{aligned}
Q\left(x_{1}+Q\left(x_{2}\right)\right) & =Q\left(x_{1}\right)+Q\left(x_{2}\right), \\
Q\left(-x_{2}\right) & =-Q\left(x_{2}\right) .
\end{aligned}
$$

We call a regular (see [4]) quantizer satisfying these conditions a quasi - uniform quantizer. We note that all uniform quantizers are quasi-uniform. In addition to assuming a quasiuniform quantzier, the approach in [1] requires all coefficients $x_{i}$ to be quantized with the same step size.

In particular for audio coding applications, both assumptions are unrealistic. First, several audio codecs, such as AC-3 
[5], routinely use uniform quantizers having different quantization step sizes for different frequency domain MDCT coefficients. Thus, although it is useful to correlate different quantized MDCT coefficients from different frequency regions in order to increase error robustness and sound quality, this would be impossible without adding distortion when using (1) and (2). Second, other common audio codecs such as MPEG-4 $\mathrm{AAC}$ [6] use quantizers that are not quasi-uniform.

This paper addresses these shortcomings of the existing theory of multiple description and proposes generalized correlating and decorrelating transforms that do not add additional distortion to the signal.

Sec. 2 describes the first contribution of this paper, a noiseless transform which permits the correlation of coefficients that are quantized using the same quasi-unform quantizer and applying different quantization step sizes for different coefficients. Sec. 3 describes the second contribution of this paper, a more complex type of transform that permits to correlate coefficients quantized by any quantizer using different quantization step sizes for each coefficient.

The third contribution addresses the high computational complexity of the correlation transform (5). The complexity derives from the involved matrix multiplications and the subsequent rounding operations. The complexity is particularly impractical for hierarchical multiple description schemes as described in [1]. For specific correlating transforms, in sec. 4 we present an application of the Hadamard matrix that significantly reduces the complexity of the transforms in hierarchical multiple description systems.

\section{NOISELESS TRANSFORMS FOR QUASI-UNIFORM QUANTIZERS}

In this sec., we introduce transforms that allow noiseless correlation and decorrelation of $G$ coefficients $x=\left(x_{i}\right)_{1 \leq i \leq G}$. We consider a quantizer $Q$ and define $O=\left(Q_{i}\right)_{1 \leq i \leq G}$ as the finite set of quantization step sizes of $Q$. Thus, $Q_{i}\left(x_{i}\right)$ is the value of $x_{i}$ after quantization by $Q$ with the step size $Q_{i}$. We assume that each coefficient $x_{i}$ has been quantized by $Q$ with the stepsize $Q_{i}$, i.e., $x_{i}=Q_{i}\left(x_{i}\right), 1 \leq i \leq G$.

For a matrix $T=T_{1} . . T_{k}$ with determinant equal to 1 , we define the correlating and decorrelating transforms $D_{T, O}(x)=$ $y$ and $D_{T^{-1}, O}(y)=x$ as follows:

$$
\begin{aligned}
D_{T, O}(x) & =C_{T_{1}, O}\left(C_{T_{2}, O}\left(\ldots C_{T_{k}, O}(x) \ldots\right)\right) \\
D_{T^{-1}, O}(y) & =C_{T_{k}^{-1}, O}\left(C_{T_{k-1}^{-1}, O}\left(\ldots C_{T_{1}^{-1}, O}(y) \ldots\right)\right),(6)
\end{aligned}
$$

where we define the operator $C_{T, O}: \mathbb{R}^{G} \rightarrow \mathbb{R}^{G}$ as

$$
y=C_{T, O}(x), \quad y_{i}=Q_{i}\left(\sum_{j=1}^{D} \tilde{t}_{i, j} x_{j}\right)
$$

with $y=\left(y_{1}, \ldots, y_{G}\right)^{t r}, T=\left(\tilde{t}_{i, j}\right)_{1 \leq i, j \leq G}$, and $C_{T^{-1}, O}$ is defined accordingly.
We now show that the transforms $D_{T, O}(x)$ and $D_{T^{-1}, O}(x)$ allow a perfect recovery of the vector $x$ if all descriptions are received at the receiver. To do so, it is sufficient to show that for a vector $x$ where each of its components $x_{i}$ has already been quantized with quantization step size $Q_{i}$, there is

$$
C_{T_{i}^{-1}, O}\left(C_{T_{i}, O}(x)\right)=x, 1 \leq i \leq k .
$$

We assume that for $T_{i}=\left(t_{i, j}\right)_{1 \leq i, j \leq G}$ there is $t_{i, j}=0$ if $i \neq j$ with the exception of $t_{i_{0}, j_{0}}$, say. Then by (4)

$$
\begin{aligned}
& C_{T_{i}, O}(x) \\
= & \left(x_{1}, . ., x_{i_{0}-1}, Q_{i_{0}}\left(x_{i_{0}}+t_{i_{0}, j_{0}} x_{j_{0}}\right), x_{i_{0}+1}, . ., x_{G}\right) \\
= & \left(x_{1}, . ., x_{i_{0}-1}, x_{i_{0}}+Q_{i_{0}}\left(t_{i_{0}, j_{0}} x_{j_{0}}\right), x_{i_{0}+1}, . ., x_{G}\right),
\end{aligned}
$$

and therefore by (3) and by (4) and the regularity of $Q$ :

$$
\begin{aligned}
& C_{T_{i}^{-1}, O}\left(C_{T_{i}, O}(x)\right) \\
= & \left(x_{1}, . ., Q_{i_{0}}\left(x_{i_{0}}+Q_{i_{0}}\left(t_{i_{0}, j_{0}} x_{j_{0}}\right)-t_{i_{0}, j_{0}} x_{j_{0}}\right), . ., x_{G}\right) \\
= & \left(x_{1}, . ., x_{i_{0}}+Q_{i_{0}}\left(t_{i_{0}, j_{0}}\right)+Q_{i_{0}}\left(-t_{i_{0}, j_{0}} x_{j_{0}}\right), . ., x_{G}\right) \\
= & x, \text { q.e.d. }
\end{aligned}
$$

We note that the noiseless character of the transforms $D_{T, O}$ and $D_{T^{-1}, O}$ is achieved by the specific way of applying the quantization step sizes $Q_{i}$ in each operator $C_{T, O}$.

\section{NOISELESS TRANSFORMS FOR GENERAL QUANTIZERS}

In this sec., we design a correlation scheme that allows to noiselessly correlate and de-correlate $G$ coefficients $x=\left(x_{i}\right)_{1 \leq i \leq G}$ that are quantized using any, not necessarily quasi-uniform quantizer $P$. The basic idea of this correlation scheme is to pre-process the input coefficients with an invertible function so that the outputs of the function are all quasi-uniformly quantized. The outputs are then correlated using the correlating transform described in sec. 2. At the decoder, the inverse mapping is performed after performing the decorrelating transform from sec 2.

Let $x_{i}$ be coefficients quantized with the quantization step sizes $P_{k}, 1 \leq k \leq H$, respectively. The finite code book $B$ of the quantizer $P$ consists of the output values $b_{k}, 1 \leq k \leq U$. By defining the mapping operator $S: B \rightarrow \Upsilon:=\mathbb{Z} \bigcap[1, U]$, as $S\left(b_{k}\right)=k$, we define a one-to-one mapping from the code book of $B$ into the set $\Upsilon$. The set $\Upsilon$ is naturally quantized by the rounding operation that maps a real number to its nearest integer. Obviously, this quantizer is quasi-linear and thus satisfies the condition of the original correlation transform proposed in [1]. Based on this observation, we propose the following transform:

1. At the sender, the mapping $S$ is applied to the coefficients $x_{i}$. The resulting integers $h_{i}, 1 \leq i \leq G, h_{i} \in \Upsilon$ are then transformed to $M D$ coefficients $J=\left(j_{1}, . ., j_{G}\right)$ using the equation (1). 
2. In case all descriptions are received at the receiver, the integers $h_{i}$ are recovered from the MD coefficients $j_{i}$ (using (2)) and the coefficients $x$ are recovered from the $h_{i}$ by inverting the map $S$.

3 . In case some $M D$ coefficients $j_{i}$ are not received, they are estimated from the received $M D$ coefficients as described in [1].

This estimation requires the knowledge of the variances of the integers $h_{i}$. We recall from the introduction that the coefficients $x_{i}=x_{i}(t)$ are measured over time $t$. Using the probability distribution of $x_{i}(t)$, one obtains the variance of $h_{i}=h_{i}(t)=S\left(x_{i}(t)\right)$. We note that a value of $h_{i}$ guessed in such a way might $\notin \Upsilon$. In this case $h_{i}$ would be set equal to the next integer $\in \Upsilon$. This approximation might introduce additional distortion when recovering the coefficients $x_{i}$.

We note that whereas the correlation scheme in sec. 2 provides noiseless recovery for all quasi-uniform quantizers, the mapping scheme in 3 allows noiseless recovery for all quantizers. Its drawbacks are the additional complexity due to the introduction of the mapping function $S$ and the potential introduction of additional distortion if some $M D$ coefficients are not received as described above.

\section{EFFICIENT CORRELATING TRANSFORMS FOR HIERARCHICAL MD SYSTEMS}

\subsection{Hierarchical multiple description systems}

Hierarchical multiple description systems [1] involve the concatenation of several layers of correlating transforms such as those given in (1), where the output of each transform layer is the input for the subsequent transform layer. These systems are useful in increasing and balancing the degree of correlation among the $M D$ coefficients.

A hierarchical $M D$ system contains $N$ initial transform

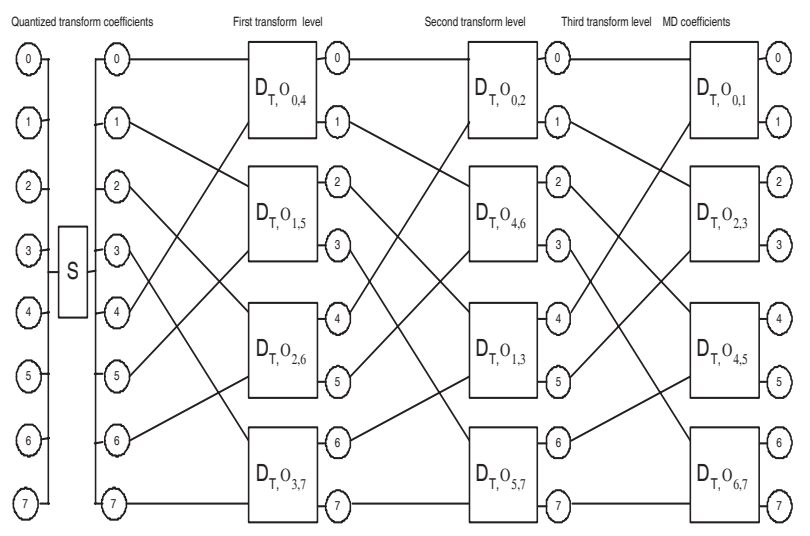

Fig. 1. A hierarchical multiple description system with $L=$ 3 and a mapping operator $S$. Using the operator $D_{T}$, two coefficients from the $n-1$-th transform level are correlated to create two coefficients of the $n$-th transform level.

coefficients, $L$ transform levels, and the $N$ coefficients are di- vided into groups of size $G=2^{L}$. Within each group, at each level of the hierarchy, sets of two coefficients from the previous hierarchy level are correlated.
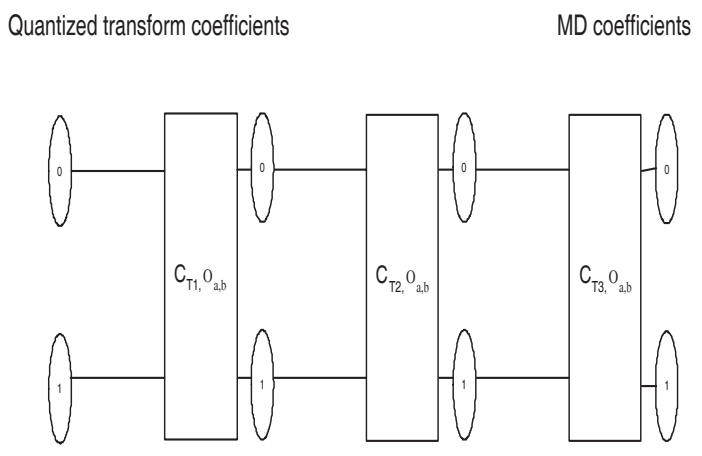

Fig. 2. Implementation of the $D_{T, O_{a, b}}$ operator.

Fig. 1 shows an example of a hierarchical multiple description system. Specifically, this hierarchical $M D$ system contains $N=8$ initial transform coefficients and $L=3$ transform levels. After the $g$-th transform, each coefficient contains information about $2^{g}$ transform coefficients. Thus, each $M D$ coefficient contains information about $2^{L}$ transform coefficients. The detailed implementation of an operator $D_{T, O_{a, b}}$ where $T$ is a $2 \times 2$ matrix and the set of quantization step sizes $O_{a, b}$ is defined as $O_{a, b}=\left(Q_{a}, Q_{b}\right)$ is shown in fig. 2. We note that different sets of quantization step sizes $Q_{a, b}$ are applied to define the operators $D_{T, O_{a, b}}$ in different parts of the hierarchical multiple description system in fig. 1.

The hierarchical multiple description system in fig. 1 applies the mapping operator $S$ defined in sec. 3 and is thus applicable to any quantizer. We note that hierarchical multiple description schemes could also be defined by correlating $M>2$ coefficients at each hierarchy level as long as $N / M^{L} \in \mathbb{Z}$.

\subsection{Efficient correlating transforms}

The practical implementation of the hierarchical multiple description system shown in fig. 1 is hindered by the successive matrix multiplication and quantization steps defining $D_{T, O}$ and $D_{T^{-1}, O}$ (see (5) and (6)). In order to reduce this computational complexity, we propose a computationally more efficient implementation of the correlating (and decorrelating) transform for hierarchical multiple description systems.

Our proposed transforms are limited to $2 \times 2$ correlating matrices $T$ defined as $\frac{1}{\sqrt{2}}\left(\begin{array}{cc}1 & 1 \\ 1 & -1\end{array}\right)$. For hierarchical multiple description systems as shown in fig. 1, these matrices are of particular importance (see [1]) because each $M D$ coefficient contains the same amount of information about any of the $2^{L}$ transform coefficients. This property is desirable when 
the different descriptions are sent over different communication channels with similar packet loss characteristics.

The proposed transform is shown in fig. 3 and consists of three steps: the mapping operator $S$ defined in sec. 3, the Hadamard transform $H_{L}$, and a scaling operator $W$. After being passed through the operator $S$, the $G$ coefficients, say $\tilde{x}=\left(\tilde{x}_{1}, . ., \tilde{x}_{G}\right)$, belonging to a given group are multiplied with the Hadamard matrix $H_{L}$ as follows:

$$
\tilde{y}=H_{L} \tilde{x}, \quad \tilde{y}=\left(y_{1}, \ldots, y_{G}\right)^{t r}
$$

where $H_{L}$ denotes the $2^{L} \times 2^{L}$ Hadamard matrix defined as

$$
H_{L}=\left(\begin{array}{cc}
H_{L-1} & H_{L-1} \\
H_{L-1} & -H_{L-1}
\end{array}\right), \quad H_{1}=\left(\begin{array}{cc}
1 & 1 \\
1 & -1
\end{array}\right) \text {. }
$$

The scaling operator $W$ is a power normalization step and corresponds to a multiplication of the coefficients $\tilde{y}_{i}$ by $2^{-L / 2}$. The complete implementation of the transform is shown in fig. 3. The decorrelating transform can be implemented in the analogous way.

Fig. 3 is an equivalent and more efficient replacement for fig. 1 when equal information about each transform coefficient is prescribed for each $M D$ coefficient in a single group, i.e., the correlation matrix $T$ is defined as above. The mathematical equivalence of the schemes in fig. 1 and 3 can be easily shown using induction but due to space constraints is not proved here. The implementation in fig. 3 is computationally significantly more efficient than the implementation in fig. 1 because of two factors:

1. No quantization steps are required for the implementation

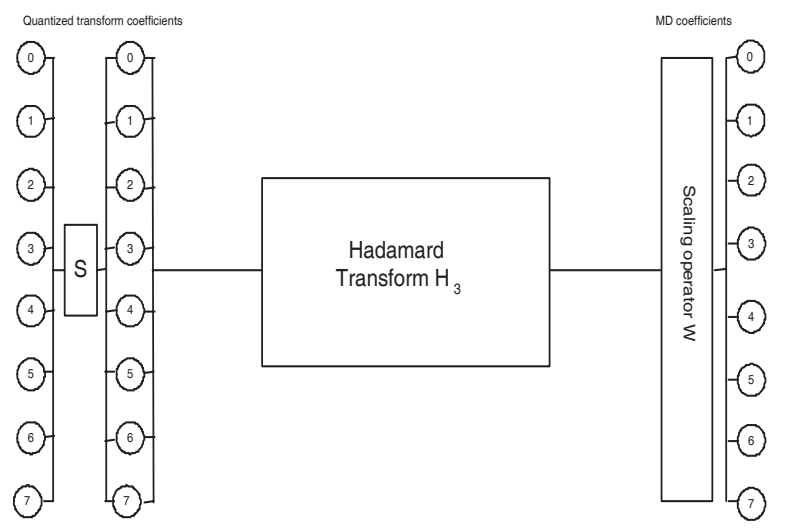

Fig. 3. Efficient implementation of a hierarchical multiple description system using the Hadamard matrix.

in fig. 3. The complexity of the operator $W$ is much lower than the complexity of the quantization operations in fig. 3 .

2. A multiplication of a vector with $G=2^{L}$ elements with the Hadamard $H_{L}$ matrix can be computed in $2^{L} L$ operations ([8]).

Table 1 gives a comparison of the number of operations for the two implementations in fig. 1 and fig. 3 as a function of the number of transform coefficients $N$ and transform levels $T$.

\begin{tabular}{|c||c|c|c|}
\hline $\mathrm{T}$ & $\begin{array}{c}\text { Orig. trans., } \\
4.5 N T \text { Ops. }\end{array}$ & $\begin{array}{c}\text { Effic. trans., } \\
\mathrm{N}(\mathrm{T}+1) \text { Ops. }\end{array}$ & $\begin{array}{c}\text { Complexity reduc. } \\
\text { of effic. trans. }\end{array}$ \\
\hline \hline 1 & 4608 & 2048 & $44.9 \%$ \\
2 & 9216 & 3072 & $48 \%$ \\
3 & 13824 & 4096 & $49.32 \%$ \\
4 & 18432 & 5120 & $49.98 \%$ \\
5 & 23040 & 6144 & $50.4 \%$ \\
6 & 27648 & 7168 & $50.6 \%$ \\
\hline
\end{tabular}

Table 1. Comparison of the number of numerical operations between the original transform, fig. 1 and the efficient transform fig. 3 for $N=1024$.

\section{CONCLUSIONS}

In this paper we extend the theory of transform based multiple description. We propose a noiseless correlation scheme that permits the correlation of coefficients quantized with the same quasi-uniform quantization scheme while using different quantization step sizes for different coefficients. We extend this transform to allow for the noiseless correlation of coefficients with any quantizer while applying different quantization step sizes to different coefficients. Finally, we use the Hadamard matrix to design an efficient and noiseless correlating transform for hierarchical multiple description schemes. The work presented here was part of a research study at Dolby Laboratories and is not intended for commercial applications.

\section{REFERENCES}

[1] Goyal, V.K., Kovačević, J., Generalized multiple description coding with correlating transforms, IEEE Trans. on Information Theory, Vol.47, No. 6, Sep. 2001.

[2] Goyal, V., Kovačević, Optimal multiple description transform coding of Gaussian vectors. Proc. IEEE Data Compression Conference 1998.

[3] Wang, Y., et al., Multiple description coding using pairwise correlating transforms, IEEE Trans. on Image Proc. 10(3): 351-366 (2001)

[4] Gersho, A., Gray, R.M., Vector Qunatization and Signal Compression, Kluwer Academic Publishers, 1992.

[5] Davidson, G.A., Digital Audio Coding: Dolby AC-3, in 'The Digital Signal Processing Handbook', V. K. Madisetti and D. B. Williams Eds., Boca Raton, Florida: CRC Press LLC, 1998, pp. 41-1 - 41-21.

[6] Bosi, M., et al., ISO/IEC MPEG-2 Advanced audio coding. Journal of the Audio engineering society, Vol. 45, No. 10, 1997, Oct, pp. 791 -811.

[7] Cheng, C., Jiang, W., Multiple description error mitigation techniques for streaming compressed audio over a 802.11 wireless network. AES 120th Conv., May 2006.

[8] Lee, M.H., Kaveh, M., Fast Hadamard transform based on a simple matrix factorization, IEEE Trans. on Acoustics, Speech, and Signal Proc., vol. ASSSP-34, no.6, pp. 1666-1667, 1986 